\title{
Modelling three dimensional gene regulatory networks
}

\author{
INNA SAMUILIK ${ }^{1}$, FELIX SADYRBAEV ${ }^{1,2,}$ \\ ${ }^{1}$ Department of Natural Sciences and Mathematics \\ Daugavpils University, Parades street1, LATVIA \\ ${ }^{2}$ Institute of Mathematics and Computer science \\ University of Latvia, Rainis boulevard 29, LATVIA
}

Abstract: We consider the three-dimensional gene regulatory network (GRN in short). This model consists of ordinary differential equations of a special kind, where the nonlinearity is represented by a sigmoidal function and the linear part is present also. The evolution of GRN is described by the solution vector $\mathrm{X}(\mathrm{t})$, depending on time. We describe the changes that system undergoes if the entries of the regulatory matrix are perturbed in some way.

Key-Words:networks, dynamical systems, attractors, gene regulation, chaos

Received: July 9, 2021. Revised: December 5, 2021. Accepted: December 17, 2021. Published: December 28, 2021.

\section{Introduction}

Under chaos in ancient Greek mythologyunderstood the pre-life mess.Greek "chaos" is the infinite firsteveryday mass, whichsubsequently gave rise to allthe existing.Physicists call this science - "nonlinear dynamics", mathematicians - "chaos theory", all the rest - "nonlinear science".

The book [14, p.310] containsone of the most popular and accepted definitions of chaos in which such systems must exhibit sensitive dependence to initial conditions, topological transitivity, and dense periodic orbits.[13] Research on chaotic systems had a practical effect since Edward Norton Lorenz established chaos theory in 1963.

Chaos should be expected to be a very common basic dynamical state in a variety of systems. Chaotic dynamics is very important in different fields such fluids, circuits, lasers, mechanical devices, chemistry, medicine (studying epilepsy to predict seizures, taking into account the initial state of the organism) and biology (in the study of uneven heart rate and unevennumber ofdiseases).[12]

Consider the general form of writing the $n$ dimensional dynamical system, that is expected to model a genetic regulatory network,

$\left\{\begin{array}{l}x_{1}^{\prime}=\frac{1}{1+e^{-\mu_{1}\left(w_{11} x_{1}+w_{12} x_{2}+\cdots+w_{1 \mathrm{n}} x_{\mathrm{n}}-\theta_{1}\right)}}-v_{1} x_{1}, \\ x_{n}^{\prime}=\frac{1}{1+e^{-\mu_{\mathrm{n}}\left(w_{\mathrm{n} 1} x_{1}+w_{\mathrm{n} 2} x_{2}+\cdots+w_{\mathrm{nn}} x_{\mathrm{n}}-\theta_{\mathrm{n}}\right)}-v_{\mathrm{n}} x_{\mathrm{n}}}\end{array}\right.$

where $\mu_{i}>0, \theta_{i}$ and $v_{i}>0$ are parameters, and $w_{i j}$ are elements of the $n \times n$ regulatory matrix $W$. The parameters of the GRN have the following biological interpretations:

$v_{i}$ - the rate of degradation of the $i$-th gene expression product;

$w_{i j}$ - the connection weight or strength of control of gene $j$ on gene $i$. Positive values of $w_{i j}$ indicate activating influences while negative values define repressing influences;

$\theta_{i}$-theinfluence of external input on gene $i$, which modulates the gene's sensitivity of response to activating or repressing influences.[10]The sigmoidal function $f(z)=$ 
$\frac{1}{1+e^{-\mu}}$ is used in (1). Sigmoidal functions are monotonically increasing from zero to unity and have a single inflection point. They are many, but the above function suits well for the analysis and visualizations. A set of coefficients $w_{i j}$ form the so called regulatory matrix

$$
W=\left(\begin{array}{ccc}
w_{11} & \ldots & w_{1 n} \\
\ldots & \ldots & \ldots \\
w_{n 1} & \ldots & w_{n n}
\end{array}\right)(2)
$$

\section{Three-element GRN}

Consider the three-dimensionalsystem

$$
\left\{\begin{aligned}
x^{\prime} & =\frac{1}{1+e^{-\mu_{1}\left(w_{11} x_{1}+w_{12} x_{2}+w_{13} x_{3}-\theta_{1}\right)}}-v_{1} x_{1}, \\
x^{\prime}{ }_{2} & =\frac{1}{1+e^{-\mu_{2}\left(w_{21} x_{1}+w_{22} x_{2}+w_{23} x_{3}-\theta_{2}\right)}}-v_{2} x_{2}, \\
x^{\prime}{ }_{3} & =\frac{1}{1+e^{-\mu_{3}\left(w_{31} x_{1}+w_{32} x_{2}+w_{33} x_{3}-\theta_{3}\right)}}-v_{3} x_{3} .
\end{aligned}\right.
$$

The nullclines for the system (3) are defined by the relations

$$
\left\{\begin{array}{l}
x_{1}=\frac{1}{v_{1}} \frac{1}{1+e^{-\mu_{1}\left(w_{11} x_{1}+w_{12} x_{2}+w_{13} x_{3}-\theta_{1}\right)}} \\
x_{2}=\frac{1}{v_{2}} \frac{1}{1+e^{-\mu_{2}\left(w_{21} x_{1}+w_{22} x_{2}+w_{23} x_{3}-\theta_{2}\right)}},(4) \\
x_{3}=\frac{1}{v_{3}} \frac{1}{1+e^{-\mu_{3}\left(w_{31} x_{1}+w_{32} x_{2}+w_{33} x_{3}-\theta_{3}\right)}} .
\end{array}\right.
$$

The first nullcline is in the set

$\left\{\left(x_{1}, x_{2}, x_{3}\right): 0<x_{1}<\frac{1}{v_{1}},\left(x_{2}, x_{3}\right) \in R^{2}\right\}$,

the second nullcline is in the set

$\left\{\left(x_{1}, x_{2}, x_{3}\right): 0<x_{2}<\frac{1}{v_{2}},\left(x_{1}, x_{3}\right) \in R^{2}\right\}$,

and the third one is in the set

$\left\{\left(x_{1}, x_{2}, x_{3}\right): 0<x_{3}<\frac{1}{v_{3}},\left(x_{1}, x_{2}\right) \in R^{2}\right\}$.

Proposition 1. System (3) has at least one equilibrium (critical point). All equilibria are located in the open box

$$
\begin{gathered}
\left\{\left(x_{1}, x_{2}, x_{3}\right): 0<x_{1}<\frac{1}{v_{1}}, 0<x_{2}<\frac{1}{v_{2}}, 0<\right. \\
\left.x_{3}<\frac{1}{v_{3}}\right\}=: G .
\end{gathered}
$$

Due to the structure of the system and properties of sigmoidal functions, the vector field, defined by the system of ODE, is directed inward on the border of $G$. Therefore, it is invariant with respect to the system. [11]

\subsection{Stable equilibria}

The standard analysis of critical points can help to find stable equilibria. If the real parts of all three characteristic numbers are negative, this is the case. The system (3) with the regulatory matrix

$$
W=\left(\begin{array}{lll}
0 & 1 & 1 \\
1 & 0 & 1 \\
1 & 1 & 0
\end{array}\right)(5)
$$

can have one or two attractive critical points, depending on the choice of parameters $\mu$ and $\theta$.Generally, $n$-dimensional systems with the regulatory matrices of the form (5) can have up to two attractive critical points, as was initially proved for two-dimensional systems in [15] and then generalized to $n$-dimensional ones in [16]. The system (3) with the matrix

$$
W=\left(\begin{array}{lll}
0 & 2 & 0 \\
2 & 0 & 0 \\
0 & 0 & 1
\end{array}\right)(6)
$$

and $\mu_{1}=\mu_{2}=7, \mu_{3}=5 ; v_{1}=v_{2}=v_{3}=1$;

$\theta_{1}=0.8, \theta_{2}=1.0, \theta_{3}=0.5$, has six attractive critical points, which can be observed in Figure 1 (six intersections of red and greenwithblue, at the corners of a cube). To see this, it is helpful to observe that the three-dimensional system with the matrix (6) consists of a two-dimensional system and a single the first order equation. The two-dimensional system can be analyzed easily both by visual inspection of the plane vector field, or by standard analysis of three critical points. The side critical points are stable nodes and the middle one is a saddle. The first order equation is constructed in the way, that guarantees that the third nullcline of a three-dimensional system decomposes into three parallel planes, which can be seen in Figure 1 (in blue). The three pairs of stable critical points arise during this process. The same technique is used below for the 


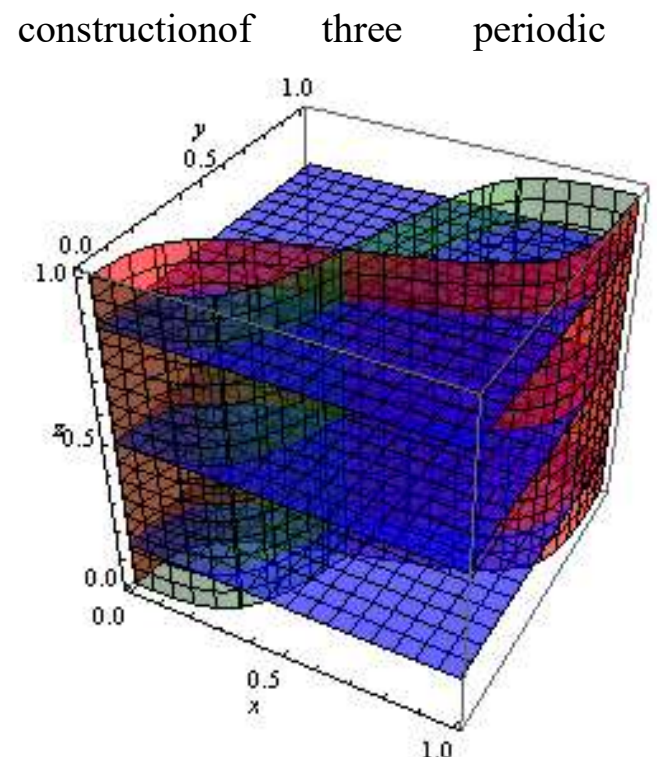

Figure 1

\subsection{Periodic attractors}

Stable limit cycles can exist in systems of the form (3). The numerically studied examples where provided in the papers [17], [18].Consider the system (3) with the matrix

$$
W=\left(\begin{array}{ccc}
1 & 0 & 2 \\
0 & 1 & 0 \\
-2 & 0 & 1
\end{array}\right)(7)
$$

and $\mu_{1}=\mu_{3}=5, \mu_{2}=15 ; \quad v_{1}=v_{2}=v_{3}=1$; $\theta_{1}=1.2, \theta_{2}=0.5, \theta_{3}=-0.6$. Three nullclines are located as shown in Figure 2.

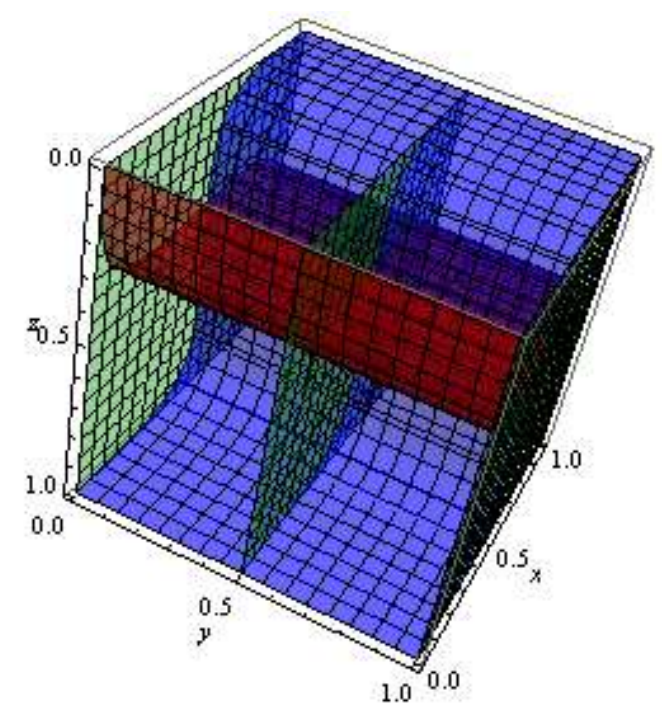

Figure 2
There are exactly three critical points $p_{1}, p_{2}$ and $p_{3}:(0.5367 ; 0.0006 ; 0.3464)$,

$(0.5367 ; 0.5 ; 0.3464)$

and

( $0.5367 ; 0.9994 ; 0.3464)$,

respectively.Linearization around these points provides us with the characteristic numbers $\lambda$ given in Table 1.

Table 1

\begin{tabular}{|c|c|c|c|}
\hline- & $\lambda_{1}$ & $\lambda_{2}$ & $\lambda_{3}$ \\
\hline $\boldsymbol{p}_{1}$ & -0.9916 & $0.1876-2.372 i$ & $0.1876+2.372 i$ \\
\hline $\boldsymbol{p}_{2}$ & 2.75 & $0.1876-2.372 i$ & $0.1876+2.372 i$ \\
\hline $\boldsymbol{p}_{3}$ & -0.9916 & $0.1876-2.372 i$ & $0.1876+2.372 i$ \\
\hline
\end{tabular}

The characteristic numbers differ only in $\lambda_{1}$. This is because the system with the regulatory matrix (7) in fact is an uncoupled system, where the twodimensional system corresponds to the first and the third rows of the matrix (7), and the second row defines one the first order equation with respect to $x_{2}$. Consequently, the second nullcline of the three-dimensional system is just a union of three planes (in green), which can be observed in Figure 2. The phase portrait for the twodimensional system is repeated three times in these $x_{2}$-nullclines. Since any time the $x_{2}$ coordinate of nullclines is changed, but other parameters no, $\lambda_{1}$ changes. Since the twodimensional system had a stable periodic solutions, all of them are in the three-dimensional phase space, depicted in Figure 3. Under the small change of parameters two side periodic trajectories become attractors, and stay attractive, while the locations of nullclines do not change significantly, but the periodic trajectory in the middle is destroyed. 


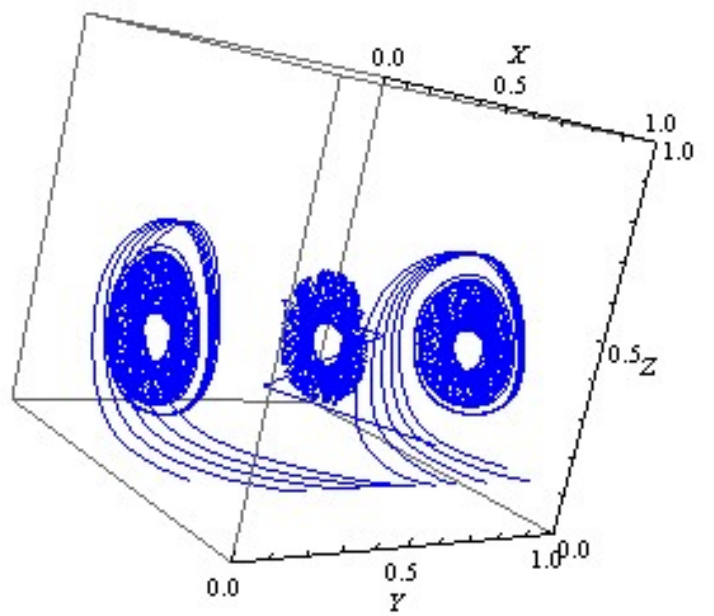

Figure 3: Three periodic solutions and some other solutions tending to the side periodic trajectories

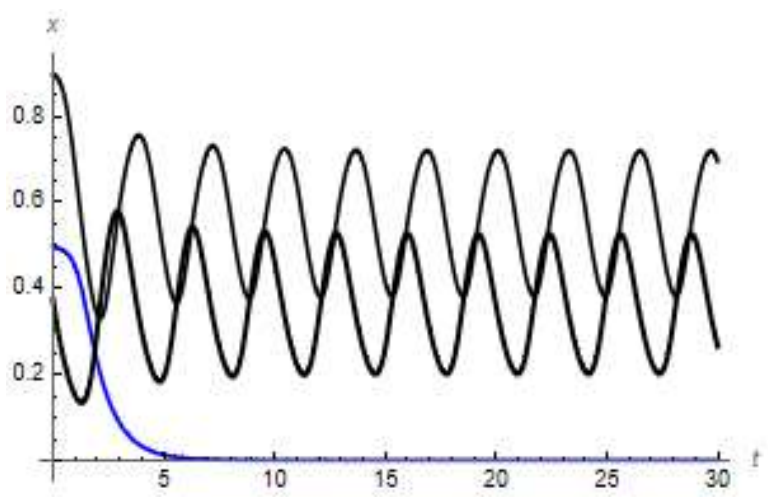

Figure 4: The graphs of $x_{i}(t), i=1,2,3$

Assume now that $w_{21}=1$ in the matrix (7). Then there is only one critical point $(0.5367 ; 0.9999998 ; 0.3464)$. The standard linearization analysis provides the characteristic numbers

$$
\lambda_{1}=-0.999997 ; \lambda_{2,3}=0.18762 \pm 2.37198 i
$$

The nullclines and one periodic solutionare depicted consequentlyin Figure 5 and Figure 6.

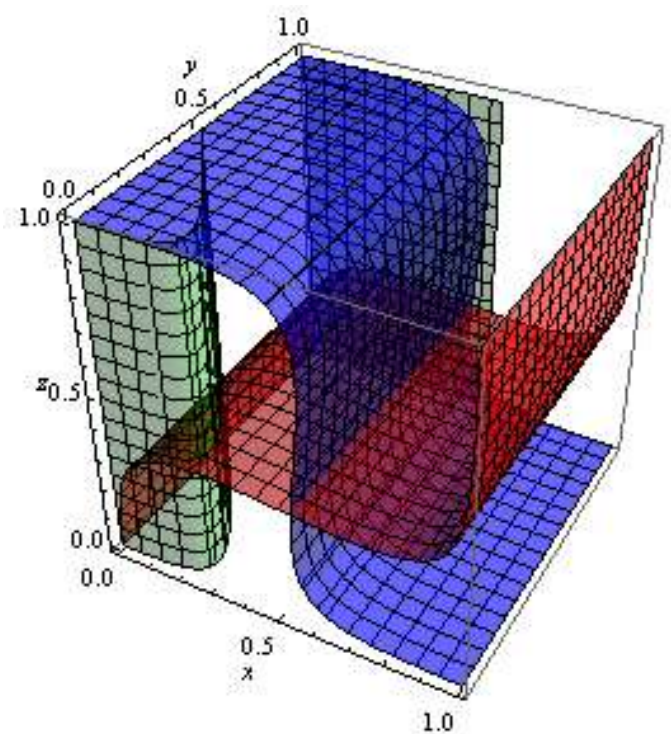

Figure 5

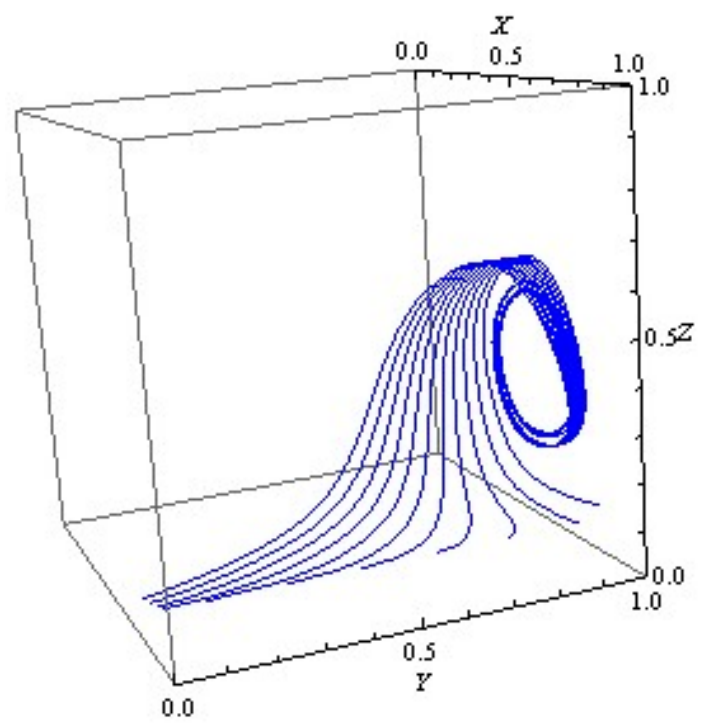

Figure 6: One periodic solution and some trajectories tending to the periodic one

\subsection{Chaotic attractors}

Chaotic attractor for the system (3) was constructed in the works [1], [2]. We consider below some modification of it.

Consider the 3-dimensional system (3) with the regulatory matrix

$$
W=\left(\begin{array}{ccc}
0 & 1 & -5.65 \\
1 & 0 & 0.135 \\
1 & 0.02 & 0.03
\end{array}\right)(8)
$$

and other parameters $\mu_{1}=\mu_{2}=7, \mu_{3}=13$; $v_{1}=0.65, v_{2}=0.42, v_{3}=0.1 ; \theta_{1}=0.5, \theta_{2}=$ $0.3, \theta_{3}=0.7$. 
The initial conditions are

$$
x_{1}(1)=0.68 ; x_{2}(1)=0.45 ; x_{3}(1)=0.15 \text {. }
$$

For this set of data, the three nullclines are located as shown in Figure 7.

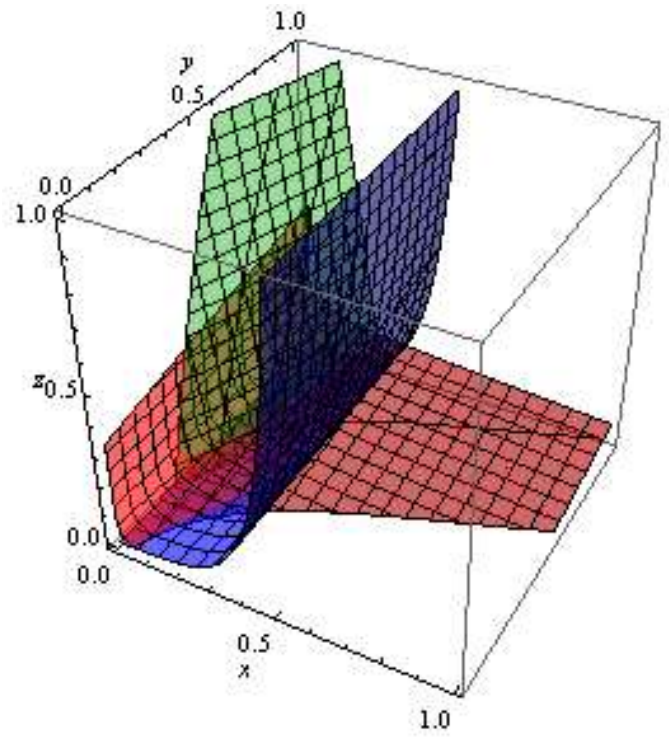

Figure 7

Thereis one critical point (0.370457; 1.59272; 0.222436). Standard linearization analysis provides the characteristic numbers

$$
\lambda_{1}=-1.2558 ; \lambda_{2,3}=0.0471391 \pm 0.739161 i
$$

The system (3) is chaotic in the sense that solutions exhibit non-regular behavior. The attractor is depicted in the figure below.

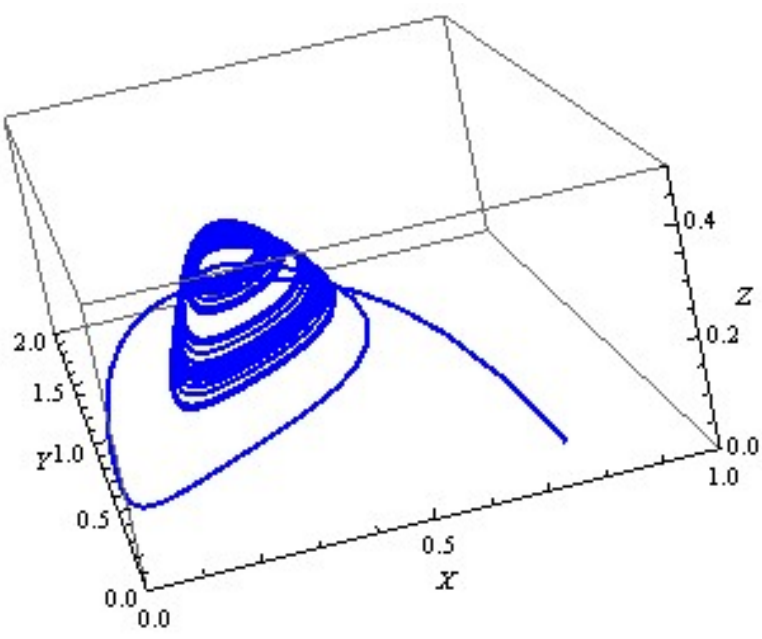

Figure 8: 3D chaotic attractor

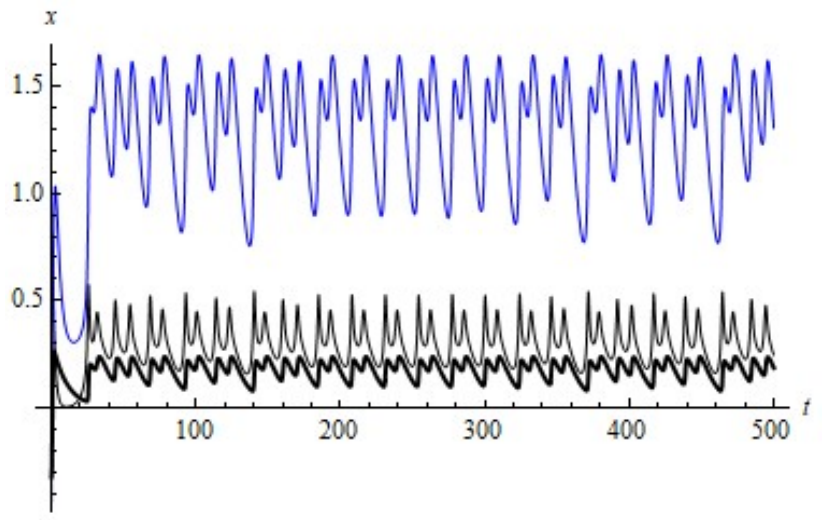

Figure 9: The graphs of $x_{i}(t), i=1,2,3$

\section{Numerical results}

Now we change the parameter $w_{23}$ (that is, the third element in the second row) in the regulatory matrix (8). The coordinates of a single critical point, values of the characteristic numbers for this point, are provided. Computations are performed using Wolfram Mathematica.

\begin{tabular}{|c|c|c|c|c|c|c|}
\hline \multirow[t]{2}{*}{$w_{23}$} & \multirow[t]{2}{*}{$x^{*}$} & \multirow[t]{2}{*}{$y^{*}$} & \multirow[t]{2}{*}{$z^{*}$} & \multirow[t]{2}{*}{ Real $\lambda$} & \multicolumn{2}{|c|}{ Complex $\lambda$} \\
\hline & & & & & $\begin{array}{l}\text { Real } \\
\text { part }\end{array}$ & $\begin{array}{l}\text { Imaginary } \\
\text { part }\end{array}$ \\
\hline 0.0 & 0.3651 & 1.4571 & 0.1989 & -1.4269 & 0.1322 & 0.6634 \\
\hline 0.05 & 0.3671 & 1.5057 & 0.2073 & -1.3714 & 0.1047 & 0.6886 \\
\hline 0.10 & 0.3691 & 1.5562 & 0.2161 & -1.3069 & 0.0726 & 0.71698 \\
\hline
\end{tabular}

Table 2 


\begin{tabular}{|l|l|l|l|l|l|l|}
\hline 0.12 & 0.3699 & 1.57699 & 0.2197 & -1.2783 & 0.0583 & 0.7294 \\
\hline 0.13 & 0.3703 & 1.5875 & 0.2215 & -1.2634 & 0.0519 & 0.7359 \\
\hline 0.132 & 0.3703 & 1.5895 & 0.2219 & -1.2604 & 0.0494 & 0.7371 \\
\hline 0.133 & 0.3704 & 1.5906 & 0.2221 & -1.2589 & 0.0487 & 0.7378 \\
\hline 0.134 & 0.3704 & 1.5917 & 0.2223 & -1.2573 & 0.0479 & 0.7385 \\
\hline 0.136 & 0.3705 & 1.5938 & 0.2226 & -1.2589 & 0.0487 & 0.7378 \\
\hline 0.137 & 0.3705 & 1.5948 & 0.2228 & -1.2527 & 0.0456 & 0.7405 \\
\hline 0.138 & 0.3706 & 1.5959 & 0.22299 & -1.2512 & 0.0448 & 0.7412 \\
\hline 0.139 & 0.3706 & 1.5969 & 0.2232 & -1.2494 & 0.0441 & 0.7418 \\
\hline 0.14 & 0.3706 & 1.59799 & 0.2234 & -1.2481 & 0.0433 & 0.7425 \\
\hline 0.145 & 0.3708 & 1.6033 & 0.2243 & -1.2403 & 0.0394 & 0.7459 \\
\hline 0.15 & 0.3710 & 1.6087 & 0.2252 & -1.2324 & 0.0354 & 0.7493 \\
\hline 0.16 & 0.3714 & 1.6192 & 0.2270 & -1.2162 & 0.0274 & 0.7564 \\
\hline 0.18 & 0.3721 & 1.6406 & 0.2308 & -1.1826 & 0.0107 & 0.7711 \\
\hline 0.19 & 0.3725 & 1.6514 & 0.2326 & -1.1652 & 0.002 & 0.7787 \\
\hline 0.20 & 0.3729 & 1.6622 & 0.2345 & -1.1473 & -0.0069 & 0.7867 \\
\hline
\end{tabular}

Calculations showed the following:

$0 \leq w_{23}<0.132$ the system (3) has a periodic solution;

$0.133<w_{23} \leq 0.137$ the system (3) is a chaotic; $0.138<w_{23} \leq 0.19$ the system (3) has a periodic solution;

$w_{23} \geq 0.2$ the system (3) is in region of asymptotic stability.

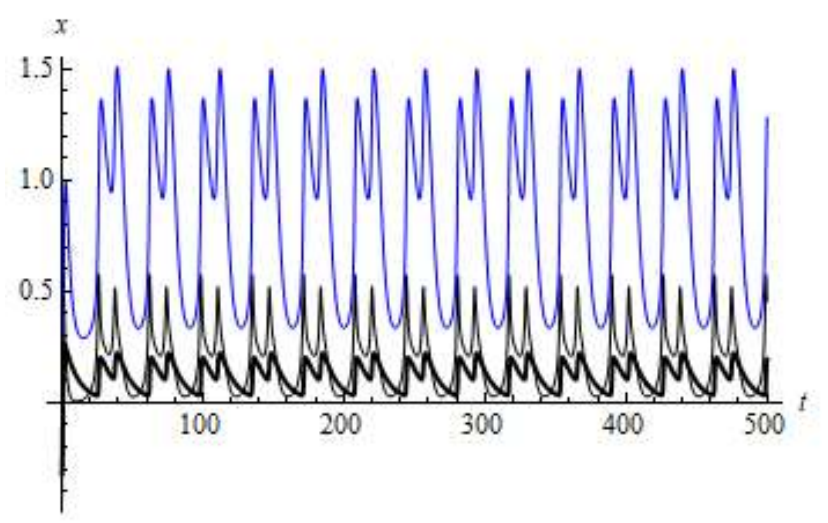

Figure 11: Solutions $x_{1}(t) ; x_{2}(t) ; x_{3}(t)$.

$$
\left(w_{23}=0.05\right)
$$




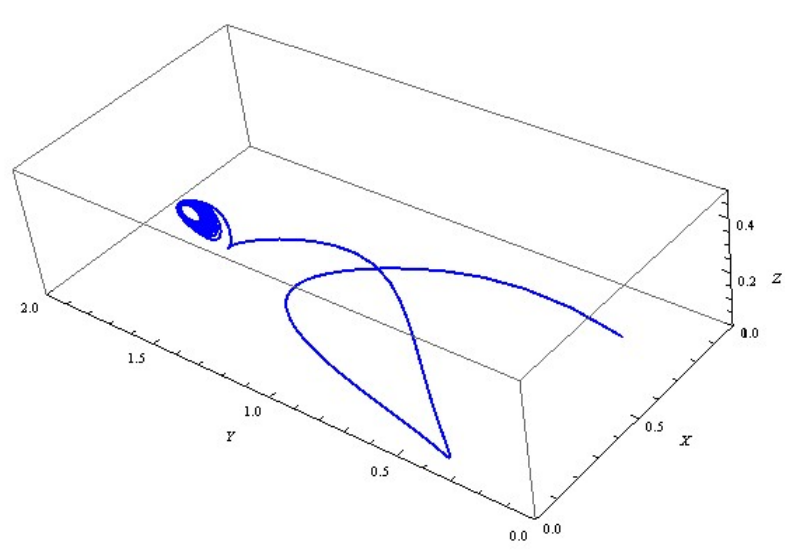

Figure 12: $w_{23}=0.19$

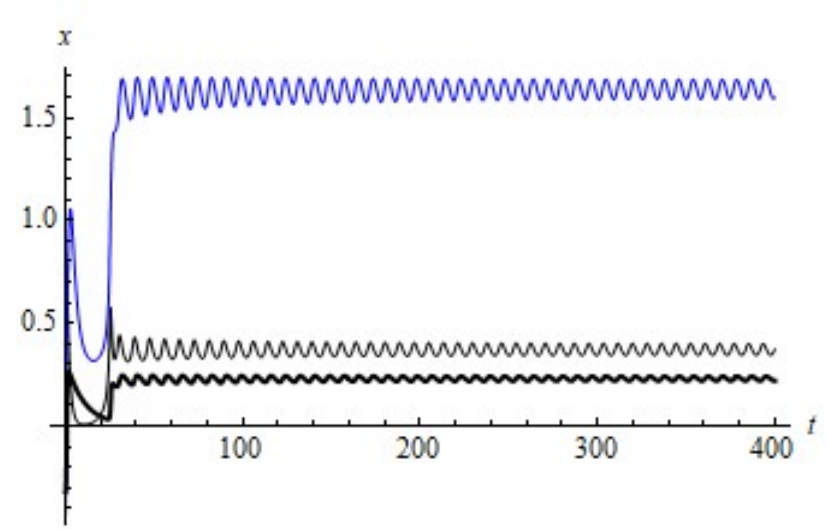

Figure 13: Solutions $x_{1}(t) ; x_{2}(t) ; x_{3}(t)$.

$$
\left(w_{23}=0.19\right)
$$

Now we change $w_{32}$ values in the regulatory matrix (8).

Table 3

\begin{tabular}{|c|c|c|c|c|c|c|}
\hline \multirow{2}{*}{$w_{32}$} & $x^{*}$ & $y^{*}$ & $z^{*}$ & Real $\lambda$ & \multicolumn{2}{|c|}{ Complex $\lambda$} \\
\cline { 5 - 7 } & & & & & $\begin{array}{c}\text { Real } \\
\text { part }\end{array}$ & $\begin{array}{c}\text { Imaginary } \\
\text { part }\end{array}$ \\
\hline 0.0 & 0.4092 & 1.7387 & 0.2449 & -1.036 & -0.0623 & 0.8666 \\
\hline 0.01 & 0.3892 & 1.6656 & 0.2337 & -1.1554 & -0.0029 & 0.7966 \\
\hline 0.03 & 0.3530 & 1.5213 & 0.2114 & -1.3366 & 0.0873 & 0.6912 \\
\hline 0.04 & 0.3368 & 1.4523 & 0.2007 & -1.3996 & 0.1186 & 0.6507 \\
\hline
\end{tabular}

From Table 3 we see, that

$0 \leq w_{32}<0.01$ the system (3) is in region of asymptotic stability;

$0.03 \leq w_{32} \leq 0.04$ the system (3) has a periodic solution.

From Table 2 and Table 3 we see that small changes in parameter values change the behavior of the system. The authors in the works [1] and [2] claim that the chaotic behavior was discovered in the system with the regulatory matrix [8] and other data given in [2]. Small variation of these data, as is shown above, keep the irregular behavior of solutions.

\section{Conclusion}

The tree-dimensional system (3) can have attractors of various kinds. It can have a single attracting point, multiple stable equilibria, but not infinite. It can have several stable periodic solutions, which serve as attractors. The irregular behavior of solutions near the chaotic attractor is possible also. It can appear in a very small diapason of parameters. The method of finding chaotic attractors is not yet developed. It is still a matter of fortune.

Chaos theory is used to explain many complex biological and natural processes. That is why, it is very important to understand at what values chaos appears. Our aim is to investigate the considered system, to find patterns of chaos.

From the practical point of view, big genomic network are responsible for reactions of living organisms to diseases like leukemia. (see [4]. [11]). The solution vector 
$X(t)=\left(x_{1}(t), \ldots, x_{n}(t)\right)$ is treated as the current state of a network, the disease is interpreted as $X(t)$ going to the "wrong" attractor. Treatment (in a model) is understood as the redirecting of a trajectory $(t, X(t))$ to a "normal" attractor. These considerations determine the future direction of research.The study of dynamical systems and

\section{References:}

[1] A.Das, A.B.Roy, Pritha Das. Chaos in a three dimensional neural network. Applied Mathematical Modelling, 24(2000), 511-522.

[2] A.Das, A.B.Roy, Pritha Das. (2002) Chaos in a Three Dimensional General Model of NeuralNetwork. International Journal of Bifurcation and Chaos, 12, 2271-2281. http://dx.doi.org/10.1142/S0218127402005820

[3] F. Sadyrbaev, I.Samuilik, V.Sengileyev, "On Modelling of Genetic Regulatory Networks," WSEAS Transactions on Electronics, vol. 12, pp. 73-80, 2021

[4] Le-Zhi Wang, Ri-Qi Su, Zi-Gang Huang,XiaoWang, WenXuWang,CelsoGrebogian dYing-ChengLai,Ageometricalapproachto controland control abilityofnonlineardynamicalnetworks. Nature

Communications, Volume7, Article number:11323(2016), DOI:10.1038/ncomms11323

[5] H. D. Jong. Modeling and Simulation of GeneticRegulatorySystems: A Literature Review, J. Comput Biol. 2002;9(1):67-103, DOI: 10.1089/10665270252833208

[6] Y. Koizumi et al. Adaptive Virtual NetworkTopology Control Based on Attractor

Selection.Journal of

LightwaveTechnology(ISSN: 0733-8724), Vol.28 (06/2010), Issue 11, pp.17201731DOI:10.1109/JLT.2010.2048412

[7] S. Atslega, F. Sadyrbaev, I. Samuilik. On Modelling Of Complex Networks. Engineering for Rural Development(ISSN 1691-5976), 2021, pp. 10091014. networks of various kinds is an urgent and timely task [19], [20].

ESF Project No. 8.2.2.0/20/I/003 "Strengthening of Professional Competence of Daugavpils University Academic Personnel of Strategic Specialization Branches 3rd Call"

[8] F. Sadyrbaev, I. Samuilik. Mathematical Modelling of Genetic Regulatory Networks. In: 2nd International Baku Conference on Scientific Research: The Book of Full Texts. Vol.1, Azerbaijan, Baku, 28-30 April, 2021. Baku: IKSAD GLOBAL Publications, 2021, pp.463469. ISBN 978-605-70554-6-0.

[9]F.Sadyrbaev.Planardifferentialsystemsarisin ginnetworkregulationtheory.AdvancedMathemat icalModels\&Applications, Vol.4,No.1,-

2019,pp.7078

[10] N.Vijesh, S. Kumar Chakrabarti, J. Sreekumar. Modeling of gene regulatory networks: A review. J. Biomedical Science and Engineering, 2013, 6, 223-231. http://dx.doi.org/10.4236/jbise.2013.62A027

[11] I. Samuilik, F. Sadyrbaev, "Mathematical Modelling of Leukemia Treatment", WSEAS Transactions on Computers, 20:274-281. DOI: $\underline{10.37394 / 23205.2021 .20 .30}$

[12] E. Ott, (2002). Chaos in Dynamical Systems (2nd ed.). Cambridge: Cambridge University Press. doi:10.1017/CBO9780511803260

[13] S. Effah-Poku, ${ }^{1}$ W. Obeng-Denteh, ${ }^{1}$ and I. K. Dontwi. A Study of Chaos in Dynamical Systems.Journal of MathematicsVolume 2018, Article ID 1808953, 5 pages

[14] M. W. Hirsch, S.Smale, R. L. Devaney. Differential Equations, Dynamical Systems, and An Introduction To Chaos. Elsevier/Academic Press, $2^{\text {nd }}$ Edition, 2004.

[15]S. Atslega, D. Finaskins and F. Sadyrbaev. 
On a Planar Dynamical System Arising in the Network Control Theory. Mathematical Modelling and Analysis,Vol. 21, 2016, Issue 3, 385-398.

[16] E. Brokan and F. Sadyrbaev. Attraction in ndimensional differential systems from network regulation theory. Math. Methods in the Applied Sciences, Vol. 41, Issue 17, 7498-7509.

[17] S. Atslega, E. Brokanand F.Sadyrbaev. Dynamical Models of Interrelation in a Class of Artificial Networks. In: Pinelas S., Graef J.R., Hilger S., Kloeden P., Schinas C. (eds) Differential and Difference Equations with Applications. ICDDEA 2019. Springer Proceedings in Mathematics \& Statistics, vol 333 (2020). https://doi.org/10.1007/978-3-030-56323318

[18] E. Brokan, F. Sadyrbaev. Remarks on GRN type systems. 4open,vol. 3 (2020), article number 8.https://doi.org/10.1051/fopen/2020009

[19] Maxim V. Shamolin, "Qualitative and Numerical Research of Body Motion in a Resisting Medium," WSEAS Transactions on Systems, vol. 20, pp. 232-243, 2021

[20] K. Suganya, V. Arulmozhi, "Passive Adaptive Network Fuzzy Inference Controller Based wheeled Mobile Robot," WSEAS Transactions on Systems, vol. 19, pp. 284-294, 2020

\section{Contribution of individual authors to the creation of a scientific article (ghost writing policy)}

Allauthorshavecontributedequallyto creation nofthisarticle.

\section{Sources of funding for research} presented in a scientific article or scientific article itself

There is no funding for this article.

\section{Creative Commons Attribution License} 4.0

\section{(Attribution 4.0 International, CC BY}

4.0)

This article is published under the terms of the CreativeCommons Attribution License 4.0 https://creativecommons.org/licenses/by/4.0/deed .en_US 